\title{
ERCC1 as predictive biomarker to platinum- based chemotherapy in adrenocortical carcinomas
}

\author{
Valeria Laufs', Barbara Altieri',2, Silviu Sbiera', Stefan Kirchere,4, Sonja Steinhauer', Felix Beuschlein ${ }^{5,6}$, \\ Marcus Quinkler ${ }^{7}$, Holger S Willenberg ${ }^{8}$, Andreas Rosenwald ${ }^{3,4}$, Martin Fassnacht ${ }^{1,4}$ and Cristina L Ronchi ${ }^{1}$ \\ 'Division of Endocrinology and Diabetes, Department of Internal Medicine I, University Hospital, University of Wuerzburg, Wuerzburg, \\ Germany, ${ }^{2}$ Division of Endocrinology and Metabolic Diseases, Institute of Medical Pathology, Catholic University of the Sacred Heart, \\ Rome, Italy, ${ }^{3}$ Institute of Pathology, University Hospital Wuerzburg, Wuerzburg, Germany., ${ }^{4}$ Comprehensive Cancer Center \\ Mainfranken, University of Wuerzburg, Wuerzburg, Germany, ${ }^{5}$ Medizinische Klinik and Poliklinik IV, Ludwig- \\ Maximilians University, Munich, Germany, ${ }^{6}$ Klinik für Endokrinologie, Diabetologie und Klinische Ernährung, \\ Universitätsspital Zürich, Zürich, Switzerland, ${ }^{7}$ Endocrinology in Charlottenburg, Berlin, Germany, and ${ }^{8}$ Division of \\ Endocrinology and Metabolism, Rostock University Medical Center, Germany \\ Correspondence should be \\ addressed to C L Ronchi \\ Email Ronchi_C@ukw.de
}

\begin{abstract}
Objective: Platinum-based chemotherapy (PBC) is the most effective cytotoxic treatment for advanced adrenocortical carcinoma (ACC). Excision repair cross complementing group 1 (ERCC1) plays a critical role in the repair of platinuminduced DNA damage. Two studies investigating the role of ERCC1 immunostaining as a predictive marker for the response to PBC in ACC had reported conflicting results. Both studies used the ERCC1-antibody clone $8 \mathrm{~F} 1$ that later turned out to be not specific. The aim of this study was to evaluate the predictive role of ERCC1 with a new specific antibody in a larger series of ACC.

Design and methods: 146 ACC patients with available FFPE slides were investigated. All patients underwent PBC (median cycles $=6)$, including cisplatin $(n=131)$ or carboplatin $(n=15)$, in most cases combined with etoposide $(n=144)$, doxorubicin $(n=131)$ and mitotane $(n=131)$. Immunostaining was performed with the novel ERCC1-antibody clone 4F9. The relationship between ERCC1 expression and clinicopathological parameters, as well as best objective response to therapy and progression-free survival (PFS) during PBC was evaluated.

Results: High ERCC1 expression was observed in $66 \%$ of ACC samples. During PBC, 43 patients experienced objective response $(29.5 \%), 49$ stable disease (33.6\%), 8 mixed response $(5.5 \%)$ and 46 progressive disease (31.5\%) without any relationship with the ERCC1 immunostaining. No significant correlation was also found between ERCC1 expression and progression-free survival (median 6.5 vs 6 months, $P=0.33, \mathrm{HR}=1.23,95 \% \mathrm{Cl}=0.82-2.0$ ).

Conclusion: ERCC1 expression is not directly associated with sensitivity to PBC in ACC. Thus, other predictive biomarkers are required to support treatment decisions in patients with ACC.
\end{abstract}

\section{Introduction}

Platinum-based chemotherapy (PBC) is the most effective cytotoxic treatment for advanced adrenocortical carcinoma (ACC), mostly in combination with etoposide and doxorubicin plus mitotane in the EDP-M regime (1). However, the best objective response rates remain below $30 \%$ and the impact on overall survival is not satisfying as shown in the phase III clinical trial FIRMACT (2). Similarly, other possible cytotoxic drugs such as streptozotocin (2) or gemcitabine did not show a better effectiveness (3) and no effective targeted therapies have 
emerged for ACC patients with advanced disease $(4,5,6)$. Finally, PBC as other chemotherapeutic combinations is associated with relevant toxicity. Thus, it is obvious that there is an urgent need of biomarkers that may serve to predict the response to $\mathrm{PBC}$.

Excision repair cross complementing group 1 (ERCC1) is an important member of the nucleoside excision repair pathway, which plays a critical role in the DNA repair by removing DNA covalent helix-distorting adducts caused by platinum compounds (7). ERCC1 has been demonstrated to be a predictive biomarker for platinum treatment in several cancers, such as non-small-cell lung cancer, testicular germ cell tumor, bladder cancer, pancreatic carcinoma and gastric cancer $(8,9,10,11,12)$. Two previous studies, one from our group (13) and one from France (14) investigated ERCC1 immunostaining in relationship with the response to $\mathrm{PBC}$ in a relatively small series of ACC patients ( $n=45$ and $n=33$, respectively). These two studies described a similar overall response rate to PBC (25-30\% of cases), but reported conflicting results regarding the influence of ERCC1 on sensitivity to PBC, being significant only in the first study. All the previous studies on ERCC1 immunostaining, including those on ACC, have been performed by using the monoclonal anti-mouse antibody clone 8F1. However, already some years ago, it had been suggested that this clone might be not specific, ERCC1 not being the principal antigen recognized by the $8 \mathrm{~F} 1$ antibody $(15,16)$. In fact, more recently, it has been demonstrated that the clone 8F1 immunoglobulin also recognizes the choline phosphate cytidyltransferase 1 alfa (PCYT1A), an unrelated nuclear membrane protein, involved in the metabolism of phosphatidylcholine biosynthesis (17). These findings raise doubts on previously published data using the clone $8 \mathrm{~F} 1$ to investigate ERCC1 as a predictive marker to $\mathrm{PBC}$ in several solid tumors. Finally, a new highly specific clone 4F9 has been identified and then validated $(17,18,19)$. Thus, the aim of the present study was to evaluate ERCC1 immunostaining with the new highly specific clone $4 \mathrm{~F} 9$ $(17,18)$ in a larger series of ACC and to correlate it with the response to $\mathrm{PBC}$.

\section{Subjects and methods}

\section{Patients and treatment regimen}

Inclusion criteria were age of at least 18 years, histopathologic diagnosis of ACC, available formalinfixed paraffin-embedded (FFPE) specimens and treatment with PBC. We identified a total of 153 patients who fulfilled these criteria and were treated with PBC in our centers between 2004 and 2015. Seven of these patients received only one cycle of PBC and were then excluded from further analysis. Thus, the final series included 146 patients with advanced ACC (F:M=90:56, median age 48 years). None of these patients were already included in our previous paper on ERCC1 (13), while 49 participated in the FIRM-ACT study (2). Specifically, 127 samples derived from primary surgery, 6 from local recurrences, 4 from biopsies (patients not operable) and 9 from distant metastasis. The baseline clinical parameters, such as sex, age at initial diagnosis, tumor size, biochemical evaluation, tumor stage according to the European Network for the Study of Adrenal Tumors (ENSAT) classification (20), Weiss score, Ki67 proliferation index, presence and number of distant metastases and previous local and/ or pharmacological treatments are given in Table 1. All baseline data were collected through the ENSAT Registry (www.ens@t.org/registry).

The treatment regimen included cisplatin $(n=131)$ or carboplatin $(n=15)$ and was in most cases administered as combination therapy (Table 1). The median number of PBC cycles was 6 ranging from 2 to 15. Treatment was discontinued in cases of unacceptable toxicity, patient's refusal or evidence of disease progression. A total of 131 patients (90\% of cases) were treated with concomitant mitotane (target plasma concentration: $14-20 \mathrm{mg} / \mathrm{L}$ ). 114 patients received $\mathrm{PBC}$ as first-line cytotoxic treatment $(78 \%$ of cases), while the remaining 32 patients were treated with $\mathrm{PBC}$ as second- or third-line therapy, with a history of failed streptozotocin (2) or gemcitabine+capecitabine (3) (Table 1). All patients had undergone regular and standard follow-up visits with clinical, biochemical and radiological (abdominal and thoracic CT scan with contrast agent) evaluation with a staging interval usually every 8 weeks. The sensitivity to $\mathrm{PBC}$ was evaluated as progression-free survival during treatment and as best overall objective response. For this evaluation, according to our clinical practice, all radiological images were reviewed by the local expert radiologists and discussed in our multidisciplinary tumor board meetings to determine a final consensus response (progressive disease, stable disease, partial or complete response). Clinical benefit was defined as stable disease or treatment response for a minimum of 4 months.

The collection of the clinical data and the biomaterial for this retrospective study was approved by the Ethics Committee of the University of Wuerzburg (No. 93/02 and 88/11) according to the Declaration of Helsinki. Written informed consent was obtained from all patients. 
Table 1 Clinical characteristics of the cohort of 146 patients with adrenocortical carcinomas treated with platinum-based chemotherapy (PBC).

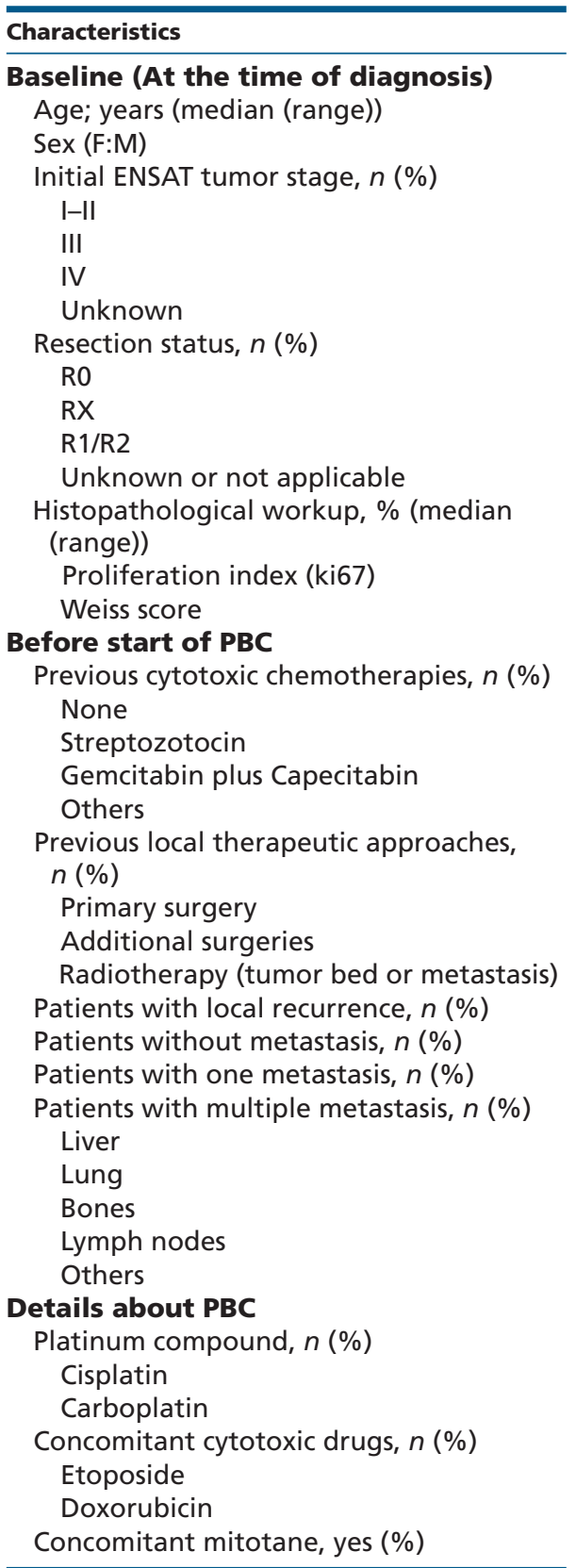

Values

$48(19-78)$ 90:56

$48(32.8 \%)$

$29(19.9 \%)$

$52(35.6 \%)$

$17(11.7 \%)$

$57(39.1 \%)$

$11(7.5 \%)$

$39(26.7 \%)$

$39(26.7 \%)$

$20(1-80)$

7 (3-9)

$114(78.1 \%)$

$26(17.8 \%)$

$4(2.7 \%)$

$2(1.4 \%)$

$141(96.6 \%)$

47 (32.2\%)

$28(19.2 \%)$

$76(52 \%)$

$1(0.7 \%)$

$15(10.3 \%)$

$130(89.0 \%)$

$102(69.9 \%)$

$114(78.1 \%)$

$32(21.9 \%)$

$77(52.7 \%)$

78 (53.4\%)

$131(89.7 \%)$

$15(10.3 \%)$

$144(99.3 \%)$

$131(90.3 \%)$

$131(90.0 \%)$

$F$, female; $\mathrm{M}$, male; $n$, number of patients; $\mathrm{PBC}$, platinum-based chemotherapy; R0, complete resection; RX, uncertain resection; R1, microscopic incomplete resection; R2, macroscopic incomplete resection.

\section{Immunohistochemistry}

A total of 146 FFPE adrenocortical tissues on standard full slides were evaluated by immunohistochemistry. In brief, sections were deparaffinized and immunohistochemical detection was performed using an indirect immunoperoxidase technique after high-temperature

antigen retrieval in $10 \mathrm{mM}$ citric acid monohydrate buffer ( $\mathrm{pH}$ 6.5) in a pressure cooker for $13 \mathrm{~min}$. Blocking of unspecific protein-antibody interactions was performed with 20\% human $A B$ serum in PBS for $1 \mathrm{~h}$ at room temperature. Primary antibody for ERCC1 was the new highly specific monoclonal anti-mouse antibody (mAb) clone 4F9 (UM500008, dilution 1:100) that was purchased from OriGene Technologies (Rockville, MD, USA). A mouse negative control was used (Dako North America). The slices were incubated overnight at $4^{\circ} \mathrm{C}$. Signal amplification was achieved with En-Vision System Labeled Polymer-HRP Anti-Mouse (Dako) for $40 \mathrm{~min}$ and developed for $10 \mathrm{~min}$ with DAB Substrate Kit (Vector Laboratories, Burlingame, CA, USA) according to the manufacturer's instructions. Nuclei were counterstained with Mayer's hematoxylin for $2 \mathrm{~min}$. For positive controls, sections of colon adenocarcinoma, renal cell carcinoma, breast cancer, hepatocellular carcinoma and normal tonsil were chosen, while cells of the tumor stroma served as internal negative control.

All slides were analyzed independently by two investigators blinded to clinical information ( $\mathrm{V}$ L and S St). Nuclear staining intensity was graded as negative (0), low (1), medium (2) or strong (3). The percentage of tumor cells with positive nuclei was calculated for each specimen and scored 0 if $0 \%$ were positive, 0.1 if $1-9 \%$ were positive, 0.5 if $10-49 \%$ were positive and 1 if $50 \%$ or more were positive. A semiquantitative $H$-score was then calculated by multiplying the staining intensity grading score with the proportion score as described previously (13). In case of discrepant results, staining intensities were jointly assessed by both investigators, forming the final score by consensus. Inter-observer agreement was investigated via Pearson's correlation coefficients 0.72 (95\% CI: 0.63-0.79).

\section{Comparison between anti-ERCC1 antibody clone 8F1 vs clone $4 \mathrm{F9}$}

We also intended to re-evaluate our old results obtained with the mAb against ERCC1 clone 8F1 (old batch) (13) with the new high specific mAb clone 4F9. To this aim, we re-stained 38 ACC samples out of the 45 previously published and re-investigated the relationship between ERCC1 expression and the response to $\mathrm{PBC}$ in terms of both progression-free survival (PFS) and disease-specific survival (DSS) after treatment. Moreover, the specificity of the currently available clone $8 \mathrm{~F} 1$ has been shown to be altered from the old clone $8 \mathrm{~F} 1(21,22)$. Then, we also evaluated a subgroup of 21 out of the 146 samples 
in our present series with the current clone 8F1 (new batch) in addition to the new clone $4 \mathrm{~F} 9$.

\section{Statistical analysis}

The Fisher's exact or the chi-square tests were used to investigate dichotomic variables, while continuous variables were investigated with a two-sided $t$ test (or non-parametric test). A non-parametric Kruskal-Wallis test, followed by Dunn's test, was used for comparison among several groups for non-normal distributed variables. Correlations and 95\% confidence intervals (95\% CI) between different parameters were evaluated by linear regression analysis. PFS was defined as the time from the date of first administration of PBC to the first radiological evidence of disease progression or death, as appropriate. DSS was defined as the time from the first administration of $\mathrm{PBC}$ to disease-specific death or last follow-up. All survival curves were obtained with KaplanMeier estimates, and the differences between survival curves were assessed by the log-rank (Mantel-Cox) test. For the calculation of hazard ratios (HR), two ACC groups with low or high protein expression were considered (high expression: $H$-score $\geq 2$ ). A multivariate regression analysis was performed via a Cox proportional hazard regression model, aiming to identify factors that might independently influence survival. Statistical analyses were made using GraphPad Prism (version 6.0) and SPSS Software (PASW, version 21.0, SPSS). $P$ values $<0.05$ were considered as statistically significant.

\section{Results}

\section{Efficacy of platinum-based chemotherapy}

The data about efficacy of PBC in the current series of 146 patients with advanced ACC are summarized in Table 2.
Concerning the best objective response during $\mathrm{PBC}$, one patient experienced complete response $(0.7 \%)$ and 42 patients partial remission $(28.8 \%), 49$ stable disease (33.5\%), 8 mixed response (5.5\%) and 46 progressive disease (31.5\%), respectively. The median PFS during PBC was 6 months, ranging from 2 to 18, while the median DSS was 17 months, ranging from 1.5 to 127 . Additionally, we observed a clinical benefit defined as at least a stable disease for a minimum of 4 months in 84 patients (58\%) with a median PFS in this group of 6 months (range: 4-18). Only one patient died unrelated to ACC during follow-up. Thus, overall survival was more or less identical to DSS (data not shown).

\section{ERCC1 expression and baseline clinical characteristics in $\mathrm{ACC}$}

Nuclear ERCC1 immunostaining was homogeneous in individual ACC samples with a median percentage of positive cells of $80 \%$ (>50\% in 135/146 samples, $92.5 \%$ ). Tissue samples exemplifying the range of staining intensity are shown in the Fig. 1. ERCC1 expression was low (H-score $0-1)$ in 50 samples (34.2\% of cases) and high (H-score 2-3) in 96 samples (65.7\%). We did not observe any significant differences in ERCC1 immunostaining among primary tumors, local recurrences and/or distant metastasis. No significant correlation was also observed between the nuclear ERCC1 expression and the ENSAT tumor stage at the time of diagnosis, the Weiss score or the Ki67 proliferation index.

\section{Predictive role of ERCC1 expression on sensitivity to platinum-based chemotherapy}

Considering the potential predictive role of ERCC1 immunostaining on the objective response to $\mathrm{PBC}$, no significant differences were observed between the

Table 2 Response to platinum-based therapy in 146 patients with adrenocortical carcinoma according to the nuclear ERCC1 immunostaining.

\begin{tabular}{|c|c|c|c|c|}
\hline & Total $(n=146)$ & Low ERCC1 $(n=50)$ & High ERCC1 $(n=96)$ & $P$ value \\
\hline \multicolumn{5}{|c|}{ Best objective response to therapy, $n(\%)$} \\
\hline Progressive disease & $46(31.5 \%)$ & $17(34 \%)$ & $29(30 \%)$ & NS \\
\hline Stable disease & $49(33.5 \%)$ & $17(34 \%)$ & $32(33 \%)$ & \\
\hline Mixed response & $8(5.5 \%)$ & $3(6 \%)$ & $5(5 \%)$ & \\
\hline Partial/Complete remission & $43(29.5 \%)$ & $13(26 \%)$ & $30(31 \%)$ & \\
\hline PFS months, median (range) & $6(1-18)$ & $6(1-15)$ & $6.5(1.5-18)$ & NS \\
\hline DSS months, median (range) & $17(1.5-127)$ & $16.5(3.5-114)$ & $17(1.5-127)$ & NS \\
\hline
\end{tabular}

www.eje-online.org 

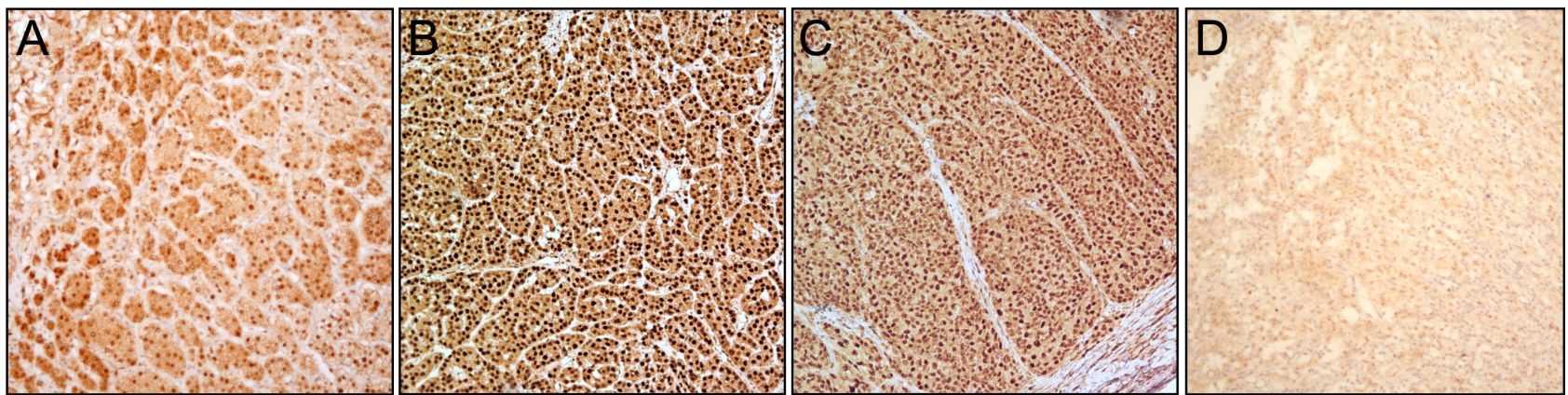

Figure 1

Representative examples of nuclear ERCC1 immunostaining in adrenocortical tissue samples using the monoclonal ERCC1 antibody clone 4F9. (A) Normal adrenal gland; (B) Adrenocortical carcinoma with high intensity and high percentage of positive cells ( $H$-score 3 ). (C) Adrenocortical carcinoma with intermediate intensity and high percentage of positive cells $(H$-score 2 ).

(D) Adrenocortical carcinoma with low intensity and low percentage of positive cells $(H$-score 0.5$)$. Magnification $1 \times 10$.

groups with high and low nuclear ERCC1 expression (Table 2). Similarly, no differences were found in terms of both PFS (median 6.5 vs 6 months, respectively, $P=0.33, \mathrm{HR}=1.23,95 \% \mathrm{CI}=0.82-2.0$ ) and DSS (median

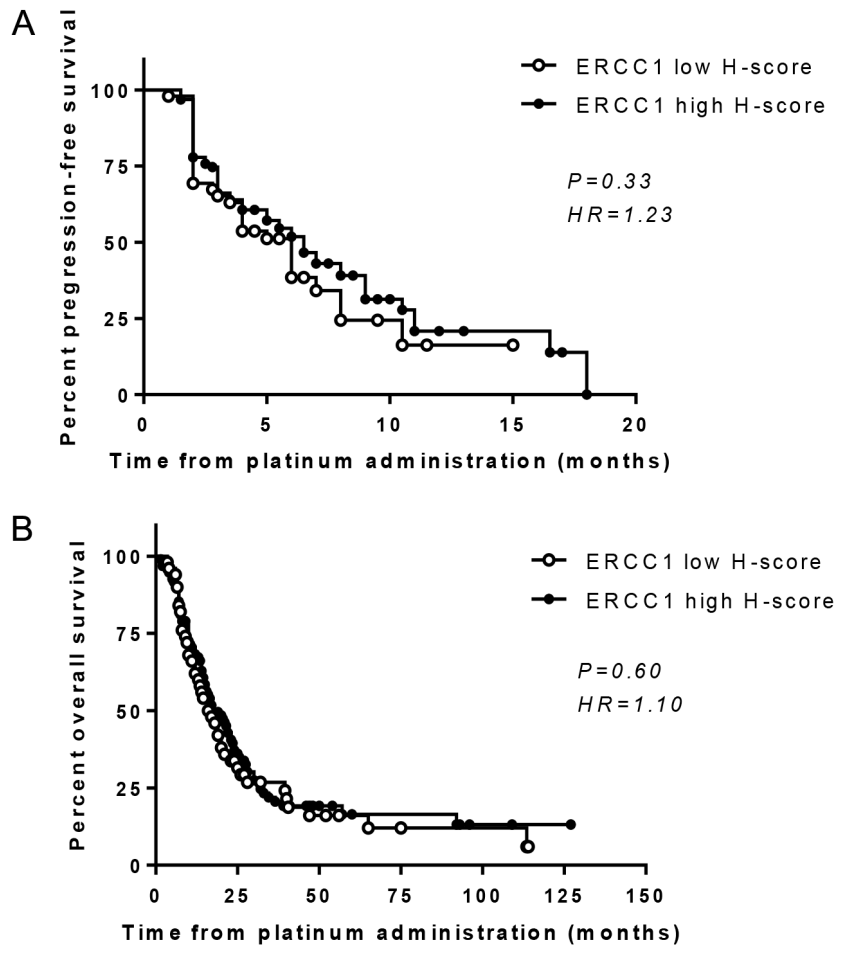

Figure 2

Relationship between ERCC1 expression and response to platinum-based chemotherapy in 146 patients with adrenocortical carcinoma (ACC). Progression-free survival (A) and overall survival $(B)$ during treatment (Kaplan-Meyer curves and log-rank test) in ACC patients with high ( $H$-score $\geq 2$ ) and low staining $(H$-score $\leq 1)$ of ERCC1.
17 vs 16.5 months, respectively, $P=0.87, \mathrm{HR}=1.03,95 \%$ $\mathrm{CI}=0.70-1.53)$ (Fig. 2A and B).

\section{Comparison between anti-ERCC1 antibody clone 8F1 vs clone 4F9}

We re-stained 38 out of 45 ACC samples of our previously published series (stained with the 8F1 clone old batch) with the new clone 4F9. Not unexpected, ERCC1 expression in terms of $H$-score corresponded in only $49 \%$ of cases. As a consequence, ERCC1 nuclear expression did no longer significantly correlate with response to $\mathrm{PBC}$ in terms of both PFS (data not shown) and DSS (Supplementary Fig. 1A and B, see section on supplementary data given at the end of this article).

Furthermore, we stained 21 out of the present 146 samples with the currently available clone 8F1 (new batch) additionally to the clone 4F9. Two representative examples are shown in the Supplementary Fig. 2. Comparing the ERCC1 immunostaining results, we observed here a correspondence between the two antibodies in $81 \%$ of cases.

\section{Discussion}

We evaluated the potential role of ERCC1 nuclear expression as predictive biomarker to $\mathrm{PBC}$ in the largest series of ACC patients up to date $(n=146)$ by using for the first time the new high ERCC1-specific monoclonal antibody clone 4F9. To note, ERCC1 has been previously demonstrated to be a predictive biomarker for platinum treatment in several cancers, such as non-small-cell lung cancer (NSCLC), testicular germ cell tumors, bladder cancer, pancreatic carcinoma and gastric cancer $(8,9,10$, $11,12)$. In ACC, we previously demonstrated in a relatively 
small series of patients that ERCC1 immunostaining was significantly correlated with overall survival during PBC (13). Another study, however, did not confirm this finding (14). Nevertheless, several concerns about the reliability of the ERCC1 immunohistochemical analysis have been raised recently. First, it has been demonstrated that the clone $8 \mathrm{~F} 1$ used in all the reported studies is not specific for $\operatorname{ERCC} 1(15,16,17)$. Specifically, the anti-ERCC1 antibody clone $8 \mathrm{~F} 1$ has been identified to also stain the PCYT1A, a phospholipid synthesis enzyme regulated by RAS $(17,23)$ with no known clinical implication in platinum drug resistance. PCYT1A has also been confirmed to play a role as prognostic biomarker in both lung and head and neck squamous cell carcinomas (23).

Moreover, the batch of the clone $8 \mathrm{~F} 1$ in use since 2011 seems not to be identical with the batch in use in 2006 (19), thus rendering new data about NSCLC not comparable with previous ones (22). According to this new information, important previous results on the role of ERCC1 in the treatment of NSCLC (8) have been revised by the same group (21). Furthermore, this year the first randomized trial to evaluate ERCC1 prospectively in 648 patients with NSCLC (ET trial) has been published definitively demonstrating that selecting chemotherapy using the commercially available ERCC 1 antibodies (clone 8F1) does not confer any additional survival benefit (24).

In parallel, a new highly ERCC1-specific clone 4F9 has been recently proposed and validated $(17,18,19)$. For all these reasons, we decided to use the clone $4 \mathrm{~F} 9$ to investigate a new large series of ACC samples in order to re-evaluate our previous results on ERCC1 as predictive marker of sensitivity to PBC. Most importantly, we could not confirm the previous results and our data now indicate that ERCC1 itself is probably not the main factor involved in the response to PBC in ACC patients. In addition, we were able to demonstrate that the current version of the clone $8 \mathrm{~F} 1$ significantly differs from the old one that we used for our pilot study (13), and we were not able to reproduce the earlier results using the same tumor samples.

One reason that could explain the lack of correlation between ERCC1 and PBC, independently from the issues with immunohistochemistry, is that ERCC1 works together with the XPF protein, codified by ERCC4. ERCC1-XPF complex is a two subunit structure-specific endonuclease that plays a key role during the nucleotide excision repair (NER) process $(7,25)$. Thus, XPF itself might be involved in the sensitivity to the response to PBC $(26,27)$. However, the ET trial demonstrated that XPF expression is not predictive for response to 648 patients with NSCLC (24). Moreover, the ERCC1-XPF complex makes incisions on the damaged DNA strand on the 5' side and acts in cooperation with several other proteins, such as XPC-RAD23B, XPA, RPA, TFIIH and XPG, during the NER process $(28,29)$. Thus, although ERCC1 plays a major role in the NER, several other proteins and mechanisms could influence the response to PBC.

Another explanation, why ERCC1 expression and clinical outcome in our and other series did not correlate could be the fact that virtually all patients have received in parallel to the platinum derivate 1-3 other additional cytotoxic drugs (mostly doxorubicin, etoposide and mitotane) diluting the hypothesized correlation. Other potential biomarker could for instance be involved in the prediction of response to these concomitant treatments (i.e. TOP2A (30)). Finally, one potential limitation in our study as well as in several others might be that ERCC1 was assessed on tumor specimens obtained months or even years before the start of chemotherapy. Nevertheless, we did not observe any significant differences in ERCC1 immunostaining among primary tumors, local recurrences and/or distant metastasis, thus suggesting that the ERCC1 levels remain quite stable over the time and tumor progression.

More generally, the search for predictive biomarkers to conventional cytotoxic chemotherapy has been proven challenging due to frequent discrepant and non-replicable findings. And this is true not only for protein expression where issues with antibodies and immunohistochemical analysis are common, but also for gene expression. Thus, if a plethora of biomarkers predicting chemotherapy efficacy have been evaluated in the clinical setting also, none of them is ready for clinical implementation yet (31). Considering that most mechanisms of resistance or sensitivity to chemotherapy are multifactorial, a combinatorial approach and further efforts are required (32).

Concerning the response rate to $\mathrm{PBC}$ in general, we observed an objective partial response in $29.5 \%$ of cases and a stable disease in further $33.5 \%$, thus confirming that $\mathrm{PBC}$ is currently the most effective cytotoxic therapy for advanced ACC. These data are generally superimposable to those reported in the FIRM-ACT study on EDP-M (2).

In conclusion, ERCC1 expression as detected by immunostaining is not directly associated with sensitivity to PBC in ACC. Thus, the search for predictive biomarkers in this devastating disease with poor response to medical therapy has to continue. 
Supplementary data

This is linked to the online version of the paper at https://doi.org/10.1530/ EJE-17-0788.

\section{Declaration of interest}

The authors declare that there is no conflict of interest that could be perceived as prejudicing the impartiality of this clinical study reported.

\section{Funding}

This work has been supported by the Deutsche Forschungsgemeinschaft (DFG) within the CRC/Transregio 205/1 'The Adrenal: Central Relay in Health and Disease' to M F and by an individual grant (FA466/4-1 and 4-2 to M F).

\section{Acknowledgements}

The authors are grateful to Martina Zink for excellent technical support and to Michaela Haaf for coordinating the ENSAT Registry in Wuerzburg.

\section{References}

1 Berruti A, Terzolo M, Sperone P, Pia A, Della Casa S, Gross DJ, Carnaghi C, Casali P, Porpiglia F, Mantero F et al. Etoposide doxorubicin and cisplatin plus mitotane in the treatment of advanced adrenocortical carcinoma: a large prospective phase II trial. Endocrine-Related Cancer 200512 657-666. (https://doi.org/10.1677/ erc.1.01025)

2 Fassnacht M, Terzolo M, Allolio B, Baudin E, Haak H, Berruti A, Welin S, Schade-Brittinger C, Lacroix A, Jarzab B et al. Combination chemotherapy in advanced adrenocortical carcinoma. New England Journal of Medicine 2012366 2189-2197. (https://doi.org/10.1056/ NEJMoa1200966)

3 Sperone P, Ferrero A, Daffara F, Priola A, Zaggia B, Volante M, Santini D, Vincenzi B, Badalamenti G, Intrivici C et al. Gemcitabine plus metronomic 5-fluorouracil or capecitabine as a second-/ third-line chemotherapy in advanced adrenocortical carcinoma: a multicenter phase II study. Endocrine-Related Cancer 201017 445-453. (https://doi.org/10.1677/ERC-09-0281)

4 Ronchi CL, Kroiss M, Sbiera S, Deutschbein T \& Fassnacht M. EJE prize 2014: current and evolving treatment options in adrenocortical carcinoma: where do we stand and where do we want to go? European Journal of Endocrinology 2014171 R1-R11. (https://doi. org/10.1530/EJE-14-0273)

5 Else T, Kim AC, Sabolch A, Raymond VM, Kandathil A, Caoili EM, Jolly S, Miller BS, Giordano TJ \& Hammer GD. Adrenocortical carcinoma. Endocrine Reviews 201435 282-326. (https://doi. org/10.1210/er.2013-1029)

6 Fassnacht M, Kroiss M \& Allolio B. Update in adrenocortical carcinoma. Journal of Clinical Endocrinology and Metabolism 201398 4551-4564. (https://doi.org/10.1210/jc.2013-3020)

7 Manandhar M, Boulware KS \& Wood RD. The ERCC1 and ERCC4 (XPF) genes and gene products. Gene 2015569 153-161. (https://doi. org/10.1016/j.gene.2015.06.026)

8 Olaussen KA, Dunant A, Fouret P, Brambilla E, Andre F, Haddad V, Taranchon E, Filipits M, Pirker R, Popper HH et al. DNA repair by ERCC1 in non-small-cell lung cancer and cisplatin-based adjuvant chemotherapy. New England Journal of Medicine 2006355 983-991. (https://doi.org/10.1056/NEJMoa060570)

9 Mendoza J, Martinez J, Hernandez C, Perez-Montiel D, Castro C, Fabian-Morales E, Santibanez M, Gonzalez-Barrios R, Diaz-Chavez J, Andonegui MA et al. Association between ERCC1 and XPA expression and polymorphisms and the response to cisplatin in testicular germ cell tumours. British Journal of Cancer 2013109 68-75. (https://doi. org/10.1038/bjc.2013.303)

10 Bellmunt J, Paz-Ares L, Cuello M, Cecere FL, Albiol S, Guillem V, Gallardo E, Carles J, Mendez P, de la Cruz JJ et al. Gene expression of ERCC1 as a novel prognostic marker in advanced bladder cancer patients receiving cisplatin-based chemotherapy. Annals of Oncology 200718 522-528. (https://doi.org/10.1093/annonc/mdl435)

11 Strippoli A, Rossi S, Martini M, Basso M, D’Argento E, Schinzari G, Barile R, Cassano A \& Barone C. ERCC1 expression affects outcome in metastatic pancreatic carcinoma treated with FOLFIRINOX: a single institution analysis. Oncotarget 20167 35159-35168. (https://doi.org/10.18632/oncotarget.9063)

12 De Dosso S, Zanellato E, Nucifora M, Boldorini R, Sonzogni A, Biffi R, Fazio N, Bucci E, Beretta O, Crippa S et al. ERCC1 predicts outcome in patients with gastric cancer treated with adjuvant cisplatin-based chemotherapy. Cancer Chemotherapy and Pharmacology 201372 159-165. (https://doi.org/10.1007/s00280-013-2181-2)

13 Ronchi CL, Sbiera S, Kraus L, Wortmann S, Johanssen S, Adam P, Willenberg HS, Hahner S, Allolio B \& Fassnacht M. Expression of excision repair cross complementing group 1 and prognosis in adrenocortical carcinoma patients treated with platinum-based chemotherapy. Endocrine-Related Cancer 200916 907-918. (https://doi.org/10.1677/ERC-08-0224)

14 Malandrino P, Al Ghuzlan A, Castaing M, Young J, Caillou B, Travagli JP, Elias D, de Baere T, Dromain C, Paci A et al. Prognostic markers of survival after combined mitotane- and platinum-based chemotherapy in metastatic adrenocortical carcinoma. EndocrineRelated Cancer 201017 797-807. (https://doi.org/10.1677/ERC-090341)

15 Niedernhofer LJ, Bhagwat N \& Wood RD. ERCC1 and non-small-cell lung cancer. New England Journal of Medicine 2007356 2538-2540. (https://doi.org/10.1056/NEJMc070742)

16 Bhagwat NR, Roginskaya VY, Acquafondata MB, Dhir R, Wood RD $\&$ Niedernhofer LJ. Immunodetection of DNA repair endonuclease ERCC1-XPF in human tissue. Cancer Research 200969 6831-6838. (https://doi.org/10.1158/0008-5472.CAN-09-1237)

17 Ma D, Baruch D, Shu Y, Yuan K, Sun Z, Ma K, Hoang T, Fu W, Min L, Lan ZS et al. Using protein microarray technology to screen anti-ERCC1 monoclonal antibodies for specificity and applications in pathology. BMC Biotechnology 201212 88. (https://doi. org/10.1186/1472-6750-12-88)

18 Bahamon BN, Gao F \& Danaee H. Development and validation of an ERCC1 immunohistochemistry assay for solid tumors. Archives of Pathology and Laboratory Medicine 2016140 1397-1403. (https://doi. org/10.5858/arpa.2016-0006-OA)

19 Smith DH, Fiehn AM, Fogh L, Christensen IJ, Hansen TP, Stenvang J, Nielsen HJ, Nielsen KV, Hasselby JP, Brunner N et al. Measuring ERCC1 protein expression in cancer specimens: validation of a novel antibody. Scientific Reports 20144 4313. (https://doi.org/10.1038/ srep04313)

20 Fassnacht M, Johanssen S, Quinkler M, Bucsky P, Willenberg HS, Beuschlein F, Terzolo M, Mueller HH, Hahner S, Allolio B et al. Limited prognostic value of the 2004 International Union Against Cancer staging classification for adrenocortical carcinoma: proposal for a Revised TNM Classification. Cancer 2009115 243-250. (https://doi.org/10.1002/cncr.24030)

21 Friboulet L, Olaussen KA, Pignon JP, Shepherd FA, Tsao MS, Graziano S, Kratzke R, Douillard JY, Seymour L, Pirker R et al. ERCC1 isoform expression and DNA repair in non-small-cell lung cancer. New England Journal of Medicine 2013368 1101-1110. (https://doi. org/10.1056/NEJMoa1214271)

22 Wislez M, Barlesi F, Besse B, Mazieres J, Merle P, Cadranel J, Audigier-Valette C, Moro-Sibilot D, Gautier-Felizot L, Goupil F et al. Customized adjuvant phase II trial in patients with nonsmall-cell lung cancer: IFCT-0801 TASTE. Journal of Clinical 
Oncology 201432 1256-1261. (https://doi.org/10.1200/ JCO.2013.53.1525)

23 Vaezi AE, Bepler G, Bhagwat NR, Malysa A, Rubatt JM, Chen W, Hood BL, Conrads TP, Wang L, Kemp CE et al. Choline phosphate cytidylyltransferase-alpha is a novel antigen detected by the antiERCC1 antibody 8F1 with biomarker value in patients with lung and head and neck squamous cell carcinomas. Cancer 2014120 1898-1907. (https://doi.org/10.1002/cncr.28643)

24 Lee SM, Falzon M, Blackhall F, Spicer J, Nicolson M, Chaudhuri A, Middleton G, Ahmed S, Hicks J, Crosse B et al. Randomized prospective biomarker trial of ERCC1 for comparing platinum and nonplatinum therapy in advanced non-small-cell lung cancer: ERCC1 trial (ET). Journal of Clinical Oncology 201735 402-411. (https://doi.org/10.1200/JCO.2016.68.1841)

25 Scharer OD. Nucleotide excision repair in eukaryotes. Cold Spring Harbor Perspectives in Biology 20135 a012609.

26 Olaussen KA \& Soria JC. Validation of ERCC1-XPF immunodetection - letter. Cancer Research 201070 3851-3852. (https://doi. org/10.1158/0008-5472.CAN-09-4352)

27 Kirschner K \& Melton DW. Multiple roles of the ERCC1-XPF endonuclease in DNA repair and resistance to anticancer drugs. Anti Cancer Research 201030 3223-3232.
28 Evans E, Moggs JG, Hwang JR, Egly JM \& Wood RD. Mechanism of open complex and dual incision formation by human nucleotide excision repair factors. EMBO Journal 199716 6559-6573. (https://doi.org/10.1093/emboj/16.21.6559)

29 Aboussekhra A, Biggerstaff M, Shivji MK, Vilpo JA, Moncollin V, Podust VN, Protic M, Hubscher U, Egly JM \& Wood RD. Mammalian DNA nucleotide excision repair reconstituted with purified protein components. Cell 199580 859-868. (https://doi.org/10.1016/00928674(95)90289-9)

30 Roca E, Berruti A, Sbiera S, Rapa I, Oneda E, Sperone P, Ronchi CL, Ferrari L, Grisanti S, Germano A et al. Topoisomerase 2alpha and thymidylate synthase expression in adrenocortical cancer. EndocrineRelated Cancer 201724 299-307. (https://doi.org/10.1530/ERC-17-0095)

31 Bergot E, Levallet G, Campbell K, Dubois F, Lechapt E \& Zalcman G. Predictive biomarkers in patients with resected nonsmall cell lung cancer treated with perioperative chemotherapy. European Respiratory Review 201322 565-576. (https://doi. org/10.1183/09059180.00007113)

32 Olaussen KA \& Postel-Vinay S. Predictors of chemotherapy efficacy in non-small-cell lung cancer: a challenging landscape. Annals of Oncology 201627 2004-2016. (https://doi.org/10.1093/annonc/ mdw321)

Received 25 September 2017

Revised version received 21 November 2017

Accepted 28 November 2017 